T. OTSUKI

KODAI MATH. J.

1 (1978), 237-257

\title{
CERTAIN INTEGRAL EQUALITY AND INEQUALITY FOR HYPERSURFACES OF $\boldsymbol{S}^{n}(R)$
}

\author{
By Tominosuke OTsuki
}

\section{$\S 0$. Introduction.}

We have the following well known isoperimetric inequality for any simply connected domain $\Omega$ in the sphere $S^{2}(R)$ of radius $R$ with smooth boundary $\partial \Omega$ :

$$
\begin{gathered}
\text { Let } A=\operatorname{area}(\Omega) \text { and } L=\operatorname{length}(\partial \Omega), \text { then } \\
L^{2} \geqq A\left(4 \pi-\frac{1}{R^{2}} A\right)
\end{gathered}
$$

and the equality is true if and only if $\Omega$ is a geodesic circular disk.

We can prove this inequality by a method of the integral geometry in which for any integer $k$ and positive real number $r$, the set of points $y$ of $S^{2}(R)$ such that the spherical circle with center at $y$ and of radius $r$ intersects $\partial \Omega$ at $k$ points are used effectively. In the present paper, the author will try to get analogous results to this fact in a higher dimensional sphere $S^{n}(R)$ by means of the same way.

In $\S 1$, we state some preliminary facts. In $\S 2$, we shall obtain an integral equality for oriented hypersurfaces (Theorem 1 ). Then, in $\S 3$, we shall have an equality on the volumes of a convex domain and the $r$-neighborhood $\Omega_{r}$ of $\Omega((3 \cdot 5))$. Finally, in $\S \S 4$ and 5 , combining the results in $\S \S 2$ and 3 and using the Fenchel-Borsuk's theorem:

For any closed curve $C$ in a Euclidean space, $\int_{C}|k(s)| d s \geqq 2 \pi$, where $k(s)$ is the curvature of $C$ and $s$ denotes the arclength of $C$, we shall obtain a kind of isoperimetric inequality for a convex domain in $S^{3}(R)$ (Theorem 3 ).

\section{$\S 1$. Preliminaries.}

Let $S^{n}(R)$ be the standard $n$-sphere in $R^{n+1}$ of radius $R$ and with its centor at the origin, and $\Omega$ a domain in $S^{n}(R)$ with smooth boundary $\partial \Omega=M^{n-1}(=M)$. Let $\Phi=\left\{\xi\left|\xi \in T_{x} S^{n}(R), x \in M,\right| \xi \mid=1\right\}$ and $\pi: \Phi \rightarrow M$ be the projection of the sphere bundle $(\Phi, M, \pi)$. For a positive real number $r>0$, let $\phi_{r}$ be the mapping $\phi_{r}: \Phi \rightarrow S^{n}(R)$ with $\psi_{r}(\xi)=\exp _{x} r \xi$, where $\exp _{x}$ denotes the exponential mapping of $S^{n}(R)$ at $x=\pi(\xi)$.

Received April 25, 1977. 
Let $\left(x, e_{1}, \cdots, e_{n-1}, e_{n}, e_{n+1}\right)$ be a moving orthonormal frame of $R^{n+1}$ such that

$$
x \in M, e_{1}, e_{2}, \cdots, e_{n-1} \in T_{x} M, e_{n+1}=\frac{1}{R} x
$$

and the orientation of $\left(e_{1}, e_{2}, \cdots, e_{n-1}, e_{n}, e_{n+1}\right)$ coincides with the canonical one of $R^{n+1}$. Then, we have

$$
\left\{\begin{aligned}
d x= & \sum_{\beta=1}^{n-1} \omega_{\beta} e_{\beta}, \\
d e_{\alpha}= & \sum_{\beta=1}^{n-1} \omega_{\alpha \beta} e_{\beta}+\omega_{\alpha n} e_{n}-\frac{1}{R} \omega_{\alpha} e_{n+1}, \\
& \alpha=1,2, \cdots, n-1, \\
d e_{n}=- & \sum_{\beta=1}^{n-1} \omega_{\beta n} e_{\beta}, d e_{n+1}=\frac{1}{R} \sum_{\beta=1}^{n-1} \omega_{\beta} e_{\beta}
\end{aligned}\right.
$$

and

$$
\left\{\begin{array}{l}
\omega_{\alpha \beta}=-\omega_{\beta \alpha}, \\
\omega_{\alpha n}=-\omega_{n \alpha}=\sum_{\beta=1}^{n-1} A_{\alpha_{\beta}} \omega_{\beta},
\end{array}\right.
$$

where

$$
A_{\alpha \beta}=A_{\beta \alpha}, \quad \alpha, \beta=1,2, \cdots, n-1,
$$

are the components of the 2nd fundamental form of $M$ in $S^{n}(R)$ for the unit normal vector $e_{n}$ with respect to $\left(x, e_{1}, e_{2}, \cdots, e_{n-1}\right)$.

Setting $y=\phi_{r}(\xi), \xi \in \Phi, \pi(\xi)=x$ and $\xi=\sum_{\imath=1}^{n} \xi_{\imath} e_{\imath}$, we have easily

$$
y=R\left(e_{n+1} \cos \frac{r}{R}+\xi \sin \frac{r}{R}\right)
$$

and

$$
\begin{aligned}
d y & =\cos \frac{r}{R} \sum_{\alpha=1}^{n-1} \omega_{\alpha} e_{\alpha}+R \sin \frac{r}{R} \sum_{i=1}^{n} D \xi_{\imath} e_{\imath}-\sin \frac{r}{R} \sum_{\alpha=1}^{n-1} \xi_{\alpha} \omega_{\alpha} e_{n+1} \\
& =\sum_{\alpha=1}^{n-1} \omega_{\alpha}\left(\cos \frac{r}{R} e_{\alpha}-\xi_{\alpha} \sin \frac{r}{R} e_{n+1}\right)+R \sin \frac{r}{R} \sum_{i=1}^{n} D \xi_{i} e_{\imath},
\end{aligned}
$$

where $D$ denotes the covariant differentiation $S^{n}(R)$ with respect to its Riemannian connection.

Now, when $\cos \frac{r}{R} \neq 0$ and $\sin \frac{r}{R} \neq 0$, noticing that $\cos \frac{r}{R} e_{\alpha}-\xi_{\alpha} \sin \frac{r}{R} e_{n+1}$, $\alpha=1,2, \cdots, n-1$, and $\sum_{\imath} D \xi_{\imath} e_{\imath}$ are all orthogonal to $e_{n+1} \cos \frac{r}{R}+\xi \sin \frac{r}{R}$, we obtain by a straightforward calculation 


$$
\begin{aligned}
& \left(\cos \frac{r}{R} e_{1}-\xi_{1} \sin \frac{r}{R} e_{n+1}\right) \wedge\left(\cos \frac{r}{R} e_{2}-\xi_{2} \sin \frac{r}{R} e_{n+1}\right) \wedge \cdots \\
& \wedge\left(\cos \frac{r}{R} e_{n-1}-\xi_{n-1} \sin \frac{r}{R} e_{n+1}\right) \wedge \sum_{\imath} D \xi_{\imath} e_{\imath} \wedge\left(\cos \frac{r}{R} e_{n+1}+\sin \frac{r}{R} \xi\right) \\
& \quad=\left(\cos \frac{r}{R}\right)^{n-2} D \xi_{n}\left(e_{1} \wedge e_{2} \wedge \cdots \wedge e_{n+1}\right) .
\end{aligned}
$$

We denote the volume element of $S^{n}(R)$ by $d V_{S}$. Then from the above equalities we have

$$
\psi_{r}^{*} d V_{S}=R \sin \frac{r}{R}\left(\cos \frac{r}{R}\right)^{n-2} \omega_{1} \wedge \omega_{2} \wedge \cdots \wedge \omega_{n-1} \wedge D \xi_{n},
$$

in which we may replace $D \xi_{n}$ by $d \xi_{n}$.

Then, when $\cos \frac{r}{R}=0,(1.4)$ and (1.5) turn into

$$
y=\varepsilon R \xi, \quad \varepsilon=\sin \frac{r}{R}= \pm 1
$$

and

$$
d y=\varepsilon\left\{R \sum_{\imath=1}^{n} D \xi_{\imath} e_{\imath}-\sum_{\alpha=1}^{n-1} \xi_{\alpha} \omega_{\alpha} e_{n+1}\right\}
$$

For $\xi \in \Phi$ with $\xi_{n} \neq 0$, substituting $D \xi_{n}=-\frac{1}{\xi_{n}} \sum_{\alpha} \xi_{\alpha} D \xi_{\alpha}$ into the above equality, we get

$$
d y=\varepsilon\left\{R \sum_{\alpha} D \xi_{\alpha}\left(e_{\alpha}-\frac{1}{\xi_{n}} \xi_{\alpha} e_{n}\right)-\sum_{\alpha} \xi_{\alpha} \omega_{\alpha} e_{n+1}\right\} .
$$

Noticing $e_{\alpha}-\frac{1}{\xi_{n}} \xi_{\alpha} e_{n}, \alpha=1,2, \cdots, n-1$, and $e_{n+1}$ are all orthogonal to $\xi$, we obtain

$$
\begin{gathered}
\left(e_{1}-\frac{1}{\xi_{n}} \xi_{1} e_{n}\right) \wedge \cdots \wedge\left(e_{n-1}-\frac{1}{\xi_{n}} \xi_{n-1} e_{n}\right) \wedge e_{n+1} \wedge \xi \\
=-\frac{1}{\xi_{n}} e_{1} \wedge \cdots \wedge e_{n} \wedge e_{n+1} .
\end{gathered}
$$

Hence we have in this case

(1. $\left.6_{0}\right) \quad \phi_{r}^{*} d V_{S}=\varepsilon^{n+1} \frac{1}{\xi_{n}} R^{n-1} D \xi_{1} \wedge \cdots \wedge D \xi_{n-1} \wedge \sum_{\alpha} \xi_{\alpha} \omega_{\alpha}, \quad \varepsilon=\sin \frac{r}{R}$. 
The induced Riemannian metric on $M$ from $R^{n+1}$ is written as

$$
d s_{M}^{2}=\sum_{\alpha=1}^{n-1} \omega_{\alpha} \omega_{\alpha}
$$

and we define a natural Riemannian metric on $\Phi$ by

$$
d s_{\emptyset}^{2}:=\sum_{\alpha=1}^{n-1} \omega_{\alpha} \omega_{\alpha}+\sum_{\imath=1}^{n} D \xi_{\imath} D \xi_{\imath} .
$$

Then, their volume elements $d V_{M}$ and $d V_{\emptyset}$ are clearly given by

$$
d V_{M}=\omega_{1} \wedge \cdots \wedge \omega_{n-1}
$$

and

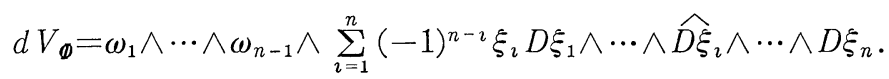

We can easily prove that the following is a differential $(n-1)$-form on $\Phi$ :

$$
d \mu_{n-1}:=\sum_{\imath=1}^{n}(-1)^{n-\imath} \xi_{\imath} D \xi_{1} \wedge \cdots \wedge \widehat{D \xi_{\imath}} \wedge \cdots \wedge D \xi_{n},
$$

whose restriction on the unit $(n-1)$-sphere $\pi^{-1}(x)$ of $T_{x} S^{n}(R), x \in M$, is its volume element. We have

$$
d V_{\emptyset}=d V_{M} \wedge d \mu_{n-1} .
$$

We can also easily prove that

$$
d \mu_{n-1}=(-1)^{n-\jmath} \frac{1}{\xi_{\jmath}} D \xi_{1} \wedge \cdots \wedge D \xi_{\jmath-1} \wedge D \xi_{j+1} \wedge \cdots \wedge D \xi_{n}
$$

at $\xi \in \Phi$ with $\xi_{j} \neq 0$, by using $\sum_{\imath} \xi_{i} \xi_{\imath}=1$ and $\sum_{\imath} \xi_{\imath} D \xi_{i}=0$, and so especially

$$
d V_{\emptyset}=\frac{1}{\xi_{n}} \omega_{1} \wedge \cdots \wedge \omega_{n-1} \wedge D \xi_{1} \wedge \cdots \wedge D \xi_{n-1}
$$

at $\xi$ with $\xi_{n} \neq 0$.

\section{§2. An integral equality for hypersurfaces $S^{n}(R)$.}

In the following, we suppose that $0<r<\pi R$. For any point $y \in S^{n}(R)$, we denote the $(n-1)$-sphere on $S^{n}(R)$ of geodesic radius $r\left(0<r<\frac{\pi}{2} R\right)$ or $\pi R-$ $r\left(\frac{\pi}{2} R \leqq r<\pi R\right)$ with its centor at $y$ or $-y$, by $F_{r}^{n-1}(y)$. We can easily see that

$$
F_{r}^{n-1}(y)=\left\{\exp _{y} v\left|v \in T_{y} S^{n}(R),\right| v \mid=r\right\}
$$


and

$$
\begin{gathered}
\phi_{r}^{-1}(y)=\left\{\text { tangent unit vectors } \xi \text { at } x \in F_{r}^{n-1}(y) \cap M\right. \\
\text { such that } \left.y=\exp _{x} r \xi\right\} .
\end{gathered}
$$

Now, when $\cos \frac{r}{R} \neq 0$, from (1.5) we have

$$
\left\{\begin{array}{l}
D \xi_{\alpha}=-\frac{1}{R} \cot \frac{r}{R} \omega_{\alpha}, \quad \alpha=1,2, \cdots, n-1, \\
D \xi_{n}=0, \\
\sum_{\alpha=1}^{n-1} \xi_{\alpha} \omega_{\alpha}=0 \quad \text { along } \quad \phi_{r}^{-1}(y) .
\end{array}\right.
$$

Hence, the induced Riemannian metric on $\psi_{r}^{-1}(y)$ from ${ }_{-}^{*}(1.8)$ on $\Phi$ can be written as

$$
d s^{2}=\left(1+\frac{1}{R^{2}} \cot ^{2} \frac{r}{R}\right) \sum_{\alpha=1}^{n-1} \omega_{\alpha} \omega_{\alpha}
$$

which implies the following equality

$$
\operatorname{vol}\left(\phi_{r}^{-1}(y)\right)=\left(1+\frac{1}{R^{2}} \cot ^{2} \frac{r}{R}\right)^{n / 2-1} \operatorname{vol}\left(F_{r}^{n-1}(y) \cap M\right) .
$$

On the other hand, we consider a differential $(n-2)$-form in $\Phi$ of the from as

$$
\Theta_{n-2}=\sum_{\alpha=1}^{n-1}(-1)^{\alpha-1} \lambda_{\alpha} D \xi_{1} \wedge \cdots \wedge \widehat{D \xi}_{\alpha} \wedge \cdots \wedge D \xi_{n-1}
$$

where $\lambda_{a}$ will be determined so that

$$
d V_{\mathscr{D}}=\psi_{r}^{*}\left(d V_{S}\right) \wedge \Theta_{n-2}
$$

By means of (1.6), where $\xi_{n} \neq 0$, the right-hand side of this equality becomes

$$
\begin{aligned}
&-R \sin \frac{r}{R}\left(\cos \frac{r}{R}\right)^{n-2} \frac{1}{\xi_{n}} \omega_{1} \wedge \cdots \wedge \omega_{n-1} \wedge \sum_{\alpha=1}^{n-1} \xi_{\alpha} D \xi_{\alpha} \\
& \wedge \sum_{\beta=1}^{n-1}(-1)^{\beta-1} \lambda_{\beta} D \xi_{1} \wedge \cdots \wedge \widehat{D}_{\beta} \wedge \cdots \wedge D \xi_{n-1} \\
&=-R \sin \frac{r}{R}\left(\cos \frac{r}{R}\right)^{n-2} \frac{1}{\xi_{n}} \sum_{\alpha=1}^{n-1} \xi_{\alpha} \lambda_{\alpha} \omega_{1} \wedge \cdots \wedge \omega_{n-1} .
\end{aligned}
$$

Comparing this with (1.13), we see that it is sufficient to take $\lambda_{\alpha}$ as 


$$
\lambda_{\alpha}=\frac{-\xi_{\alpha}}{R \sin \frac{r}{R}\left(\cos \frac{r}{R}\right)^{n-2}\left(1-\xi_{n} \xi_{n}\right)},
$$

where $\xi_{n} \xi_{n} \neq 1$.

Thus we define $\Theta_{n-2}$ by

$$
\begin{aligned}
\Theta_{n=2}:= & -\frac{1}{R \sin \frac{r}{R}\left(\cos \frac{r}{R}\right)^{n-2}} \cdot \frac{1}{1-\xi_{n} \xi_{n}} \sum_{\alpha=1}^{n-1}(-1)^{\alpha-1} \xi_{\alpha} D \xi_{1} \wedge \cdots \\
& \wedge \widehat{D \xi}_{\alpha} \wedge \cdots \wedge D \xi_{n-1},
\end{aligned}
$$

where $\xi_{n} \xi_{n}<1$.

Let $\iota_{y}: \phi_{r}^{-1}(y) \rightarrow \Phi$ be the inclusion map. Then, by (2.1) we have

$$
\begin{aligned}
c_{y}^{*} \Theta_{n-2}= & -\frac{1}{\left(R \sin \frac{r}{R}\right)^{n-1}} \cdot \frac{1}{1-\xi_{n} \xi_{n}} \sum_{\alpha=1}^{n-1}(-1)^{n-1-\alpha} \xi_{\alpha} \omega_{1} \wedge \cdots \\
& \wedge{\widehat{\omega_{\alpha}} \wedge \cdots \wedge \omega_{n-1}}, \cdots
\end{aligned}
$$

and especially

$$
\iota_{y}^{*} \Theta_{n-2}=-\frac{1}{\left(R \sin \frac{r}{R}\right)^{n-1}} \frac{1}{\xi_{n-1}} \omega_{1} \wedge \omega_{2} \cdots \wedge \omega_{n-2},
$$

where $\xi_{n-1} \neq 0$.

Next, we observe the volume element of $F_{r}^{n-1}(y) \cap M$. On $F_{r}^{n-1}(y) \cap M$, we obtain from (2.1)

$$
\begin{aligned}
d s^{2} & =\sum_{\alpha=1}^{n-1} \omega_{\alpha} \omega_{\alpha}=\sum_{a=1}^{n-2} \omega_{a} \omega_{a}+\left(-\frac{1}{\xi_{n-1}} \sum_{\alpha=1}^{n-1} \xi_{a} \omega_{a}\right)^{2} \\
& =\sum_{a, b=1}^{n-2}\left(\delta_{a b}+\frac{1}{\xi_{n-1} \xi_{n-1}} \xi_{a} \xi_{b}\right) \omega_{a} \omega_{b}
\end{aligned}
$$

and

$$
\operatorname{det}\left(\delta_{a b}+\frac{1}{\xi_{n-1} \xi_{n-1}} \xi_{a} \xi_{b}\right)=\frac{1}{\xi_{n-1} \xi_{n-1}} \sum_{\alpha=1}^{n-1} \xi_{\alpha} \xi_{\alpha}=\frac{1-\xi_{n} \xi_{n}}{\xi_{n-1} \xi_{n-1}},
$$

where $\xi_{n-1} \neq 0$. Hence, the volume element of $F_{r}^{n-1}(y) \cap M$ is given by

$$
d V_{F_{r}^{n-1}(y) \cap M}=\frac{\sqrt{1-\xi_{n} \xi_{n}}}{\xi_{n-1}} \omega_{1} \wedge \cdots \wedge \omega_{n-2},
$$


where $\xi_{n-1} \neq 0$. In general, we have

$$
d V_{F_{r}^{n-1}(y) \cap M}=(-1)^{n-1-\beta} \frac{\sqrt{1-\xi_{n} \xi_{n}}}{\xi_{\beta}} \omega_{1} \wedge \cdots \wedge \omega_{\beta-1} \wedge \omega_{\beta+1} \wedge \cdots \wedge \omega_{n-1},
$$

where $\xi_{\beta} \neq 0$, and

$$
\begin{aligned}
d V_{F_{r}^{n-1}(y) \cap M}= & \frac{1}{\sqrt{1-\xi_{n} \xi_{n}}} \sum_{\alpha=1}^{n-1}(-1)^{n-1-\alpha} \xi_{\alpha} \omega_{1} \wedge \cdots \wedge \omega_{\alpha-1} \\
& \wedge \omega_{\alpha+1} \wedge \cdots \wedge \omega_{n-1} .
\end{aligned}
$$

Since we have

$$
d V_{\psi_{r}^{-1}(y)}=\left(1+\frac{1}{R^{2}} \cot ^{2} \frac{r}{R}\right)^{n / 2-1} d V_{F_{r}^{n-1}(y) \cap M}
$$

hence

$$
\begin{aligned}
d V_{\psi_{r}^{-1}(y)}= & \left(1+\frac{1}{R^{2}} \cot ^{2} \frac{r}{R}\right)^{n / 2-1} \frac{1}{\sqrt{1-\xi_{n} \xi_{n}}} \sum_{\alpha=1}^{n-1}(-1)^{n-1-\alpha} \xi_{\alpha} \omega_{1} \wedge \cdots \\
& \wedge \omega_{\alpha-1} \wedge \omega_{\alpha+1} \wedge \cdots \wedge \omega_{n-1}
\end{aligned}
$$

and

$$
d V_{\psi_{r}^{-1}(y)}=\left(1+\frac{1}{R^{2}} \cot ^{2} \frac{r}{R}\right)^{n / 2-1} \frac{\sqrt{1-\xi_{n} \xi_{n}}}{\xi_{n-1}} \omega_{1} \wedge \cdots \wedge \omega_{n-2},
$$

where $\xi_{n-1} \neq 0$. From (2.5) and (2.8), we obtain

$$
\begin{aligned}
\iota_{y}^{*} \Theta_{n-2}= & -\frac{1}{R \sin \frac{r}{R}\left(\cos \frac{r}{R}\right)^{n-2}\left(1+R^{2} \tan ^{2} \frac{r}{R}\right)^{n / 2-1}} . \\
& \frac{1}{\sqrt{1-\xi_{n} \xi_{n}}} d V_{\psi_{r}^{-1}(y)}
\end{aligned}
$$

Finally, we consider the case $\cos \frac{r}{R}=0$, i. e. $r=\frac{\pi R}{2}$. From $\left(1.5_{0}\right)$ we have

$$
\left\{\begin{array}{l}
D \xi_{i}=0, \quad \imath=1,2, \cdots, n \\
\sum_{\alpha=1}^{n-1} \xi_{\alpha} \omega_{\alpha}=0 \quad \text { along } \phi_{r}^{-1}(y) .
\end{array}\right.
$$

We take a differential $(n-2)$-form in $\Phi$ of the form

$$
\Psi_{n-2}=\sum_{\alpha=1}^{n-1}(-1)^{\alpha-1} \lambda_{\alpha} \omega_{1} \wedge \cdots \wedge \omega_{\alpha-1} \wedge \omega_{\alpha+1} \wedge \cdots \wedge \omega_{n-1}
$$

where $\lambda_{\alpha}$ will be determined so that

$$
d V_{\emptyset}=\phi_{r}^{*}\left(d V_{S}\right) \wedge \Psi_{n-2}
$$


By means of $\left(1.6_{0}\right)$, at $\xi$ with $\xi_{n} \neq 0$, the right-hand side of this equality becomes

$$
\begin{aligned}
& \frac{1}{\xi_{n}} R^{n-1} D \xi_{1} \wedge \cdots \wedge D \xi_{n-1} \wedge \sum_{\alpha=1}^{n-1} \xi_{\alpha} \omega_{\alpha} \wedge \sum_{\beta=1}^{n-1}(-1)^{\beta-1} \lambda_{\beta} \omega_{1} \wedge \cdots \\
& \wedge \omega_{\beta-1} \wedge \omega_{\beta+1} \wedge \cdots \wedge \omega_{n-1} \\
&=(-1)^{n-1} R^{n-1} \frac{1}{\xi_{n}} \sum_{\alpha=1}^{n-1} \xi_{\alpha} \lambda_{\alpha} \omega_{1} \wedge \cdots \wedge \omega_{n-1} \wedge D \xi_{1} \wedge \cdots \wedge D \xi_{n-1}
\end{aligned}
$$

Comparing this equality with (1.13), we see that it is sufficient to take $\lambda_{\alpha}$ as

$$
\lambda_{\alpha}=\frac{(-1)^{n-1} \xi_{\alpha}}{R^{n-1}\left(1-\xi_{n} \xi_{n}\right)},
$$

when $\xi_{n} \xi_{n}<1$. Thus, we define $\Psi_{n-2}$ by

$$
\begin{aligned}
\Psi_{n-2}:= & -\frac{1}{R^{n-1}\left(1-\xi_{n} \xi_{n}\right)} \sum_{\alpha=1}^{n-1}(-1)^{n-1-\alpha} \xi_{\alpha} \omega_{1} \wedge \cdots \\
& \wedge \omega_{\alpha-1} \wedge \omega_{a+1} \wedge \cdots \wedge \omega_{n-1},
\end{aligned}
$$

where $\xi_{n} \xi_{n}<1$. In this case, from $\left(2.1_{0}\right)$ we see that

$$
d V_{\psi_{r}^{-1}(y)}=d V_{F_{r}^{n-1}(y) \cap M}
$$

through an isometry. We can also use the formula $\left(2.6^{\prime \prime}\right)$ and obtain

$$
\iota_{y}^{*} \Psi_{n-2}=-\frac{1}{R^{n-1} \sqrt{1-\xi_{n} \xi_{n}}} d V_{\psi_{r}^{-1}(y)} .
$$

Making use of these formulas and noticing that these hold good for oriented hypersurfaces in $S^{n}(R)$ in general, we obtain the following

THEOREM 1. Let $M^{n-1}=M \subset S^{n}(R)$ be a smooth oriented hypersurface and $0<r<\pi R$. Then, we have the following integral equality:

$$
\begin{gathered}
\int_{S^{n}(R)}\left(\int_{F_{r}^{n-1}(y) \cap M} \frac{1}{\sqrt{1-\xi_{n} \xi_{n}}} d V_{F_{r}^{n-1}(y) \cap M}\right) d V_{S} \\
=\left(R \sin \frac{r}{R}\right)^{n-1} \cdot c_{n-1} \operatorname{vol}(M),
\end{gathered}
$$

where $c_{n-1}$ is the volume of the unit $(n-1)$-sphere.

Proof. From (1.10), we see that

$$
\int_{\emptyset} d V_{\Phi}=\operatorname{vol}(\Phi)=c_{n-1} \cdot \operatorname{vol}(M) .
$$


We prove the case $r \neq \pi R / 2$. By means of (2.3), (2.8) and (2.7), the lefthand side of the above equality can be also computed as follows:

$$
\begin{aligned}
\int_{\Phi} d V_{\Phi}= & \int_{S^{n}(R)} \frac{d V_{S}}{R \sin \frac{r}{R}\left|\cos \frac{r}{R}\right|^{n-2}\left(1+R^{2} \tan ^{2} \frac{r}{R}\right)^{n / 2-1}} \int_{\varphi_{r}^{-1}(y)} . \\
& \frac{1}{\sqrt{1-\xi_{n} \xi_{n}}} d V_{\psi_{r}^{-1}(y)} \\
= & \frac{1}{\left(R \sin \frac{r}{R}\right)^{n-1}} \int_{S^{n}(R)}\left(\int_{F_{r}^{n-1}(y) \cap M} \frac{1}{\sqrt{1-\xi_{n} \xi_{n}}} d V_{F_{r}^{n-1}(y) \cap M}\right) d V_{S},
\end{aligned}
$$

from which we obtain immediately (2.11).

For the case $r=\pi R / 2$, we can prove it analogously by $\left(2.3_{0}\right)$ and $\left(2.9_{0}\right)$.

q. e. d.

\section{$\S 3$. An integral equality for a convex domain in $S^{3}(R)$.}

We say a domain $\Omega$ in $S^{n}(R)$ convex if $\Omega$ contains no pair of points $y$ and $-y$ of $S^{n}(R)$ and for any two points $p$ and $q$ of $\Omega$ it contains the minimum geodesic segment of $S^{n}(R)$ joining $p$ and $q$.

If $\Omega \subset S^{n}(R)$ is convex, it must be contained in a half $n$-sphere of $S^{n}(R)$. We see this fact easily by considering a contacting great $(n-1)$-sphere of $S^{n}(R)$ to $\partial \Omega$. Hence we have

$$
V=\operatorname{vol}(\Omega) \leqq \frac{c_{n}}{2} R^{n}
$$

In the following, we suppose that $\Omega$ is convex and has smooth boundary $M=\partial \Omega$. For $r>0$, we set

$$
\Omega_{r}=\left\{x \mid x \in S^{n}(R), \operatorname{dis}_{S n(R)}(x, \Omega)<r\right\}, \quad V_{r}=\operatorname{vol}\left(\Omega_{r}\right) .
$$

In this case, $M$ must be diffeomorphic to an $(n-1)$-sphere. Furthermore, we suppose $0<r \leqq \pi R / 2$. Using the notation in $\S \S 1,2$ and taking notice of that for the orthonormal frame $\left(x, e_{1}, \cdots, e_{n}, e_{n+1}\right), x \in M, e_{n}$ directs inward of $\Omega$ at $x$ and $e_{n+1}=(1 / R) x$, we see that $\Omega_{r}-\Omega$ is the set of points $y$ written as

$$
y=R\left(e_{n+1} \cos \frac{t}{R}-e_{n} \sin \frac{t}{R}\right), \quad 0 \leqq t<r .
$$


Hence, we have

$$
\begin{aligned}
d y= & \sum_{\alpha=1}^{n-1}\left\{e_{\alpha} \cos \frac{t}{R}+R\left(\sum_{\beta=1}^{n-1} A_{\alpha \beta}\right) \sin \frac{t}{R}\right\} \omega_{\alpha} \\
& -\left(e_{n+1} \sin \frac{t}{R}+e_{n} \cos \frac{t}{R}\right) d t .
\end{aligned}
$$

If we choose especially $e_{1}, \cdots, e_{n-1}$ in the principal directions of $M$ at $x$, then we can put $A_{\alpha \beta}=k_{\alpha} \delta_{\alpha \beta}$. Denoting the normal exponential map of $M$ in $S^{n}(R)$ by $\exp ^{\perp}$, we induce a volume element of the normal bundle $N M$ from $d V_{S}$ through $\exp ^{\perp}$. From the above computation, we have

$$
\left(\exp ^{\perp}\right)_{(x,-t)}^{*} d V_{s}=-\prod_{\alpha=1}^{n-1}\left(\cos \frac{t}{R}+k_{\alpha} R \sin \frac{t}{R}\right) \omega_{1} \wedge \cdots \wedge \omega_{n-1} \wedge d t
$$

hence

$$
\begin{aligned}
V_{r}-V= & \int_{0}^{r} \int_{M} \prod_{\alpha=1}^{n-1}\left(\cos \frac{t}{R}+k_{\alpha} R \sin \frac{t}{R}\right) d V_{M} d t \\
= & \int_{0}^{r} \int_{M}\left\{\left(\cos \frac{t}{R}\right)^{n-1}+\sum_{m=1}^{n-1} R^{m} \sigma_{m}\left(k_{1}, \cdots, k_{n-1}\right)\right. \\
& \left.\cdot\left(\cos \frac{t}{R}\right)^{n-m-1}\left(\sin \frac{t}{R}\right)^{m}\right\} d V_{M} d t
\end{aligned}
$$

where $\sigma_{m}\left(u_{1}, \cdots, u_{n-1}\right)$ denotes the fundamental symmetric polynomial of order $m$ in $u_{1}, u_{2} \cdots, u_{n-1}$. Thus, we have

$$
\begin{aligned}
V_{r}=V & +\int_{0}^{r}\left(\cos \frac{t}{R}\right)^{n-1} d t \cdot \int_{M} d V_{M} \\
& +\sum_{m=1}^{n-1} R^{m} \int_{0}^{r}\left(\cos \frac{t}{R}\right)^{n-m-1}\left(\sin \frac{t}{R}\right)^{m} d t \cdot \int_{M} \sigma_{m}\left(k_{1}, \cdots, k_{n-1}\right) d V_{M} .
\end{aligned}
$$

For the 2nd fundamental form $I I=\sum_{\alpha, \beta} A_{\alpha_{\beta}} \omega_{\alpha} \omega_{\beta}$, we set

$$
\operatorname{det}\left(I_{n-1}+u A\right)=1+\sum_{m=1}^{n-1}\left(\begin{array}{c}
n-1 \\
m
\end{array}\right) u^{m} P_{m}(A),
$$

where $A=\left(A_{\alpha_{\beta}}\right)$. Especially, we have

$$
P_{1}(A)=\frac{1}{n-1} \sum_{\alpha=1}^{n-1} k_{\alpha}=H \quad \text { (mean curvature). }
$$


Using these $P_{m}(A)$, we can rewrite (3.4) as

$$
\begin{aligned}
V_{r}=V & +\int_{0}^{r}\left(\cos \frac{t}{R}\right)^{n-1} d t \cdot \int_{M} d V_{M} \\
& +\sum_{m=1}^{n-1}\left(\begin{array}{c}
n-1 \\
m
\end{array}\right) R^{m} \int_{0}^{r}\left(\cos \frac{t}{R}\right)^{n-m-1}\left(\sin \frac{t}{R}\right)^{m} d t \cdot \int_{M} P_{m}(A) d V_{M} .
\end{aligned}
$$

Now, we compute the right-hand side of (3.4) in more exact form for the case $n=3$. Since we have

$$
\begin{aligned}
& \int_{0}^{r} \cos ^{2} \frac{t}{R} d t=\frac{1}{2}\left(R \cos \frac{r}{R} \sin \frac{r}{R}+r\right), \int_{0}^{r} \cos \frac{t}{R} \sin \frac{t}{R} d t=\frac{R}{2} \sin ^{2} \frac{r}{R}, \\
& \int_{0}^{r} \sin ^{2} \frac{t}{R} d t=\frac{1}{2}\left(-R \cos \frac{r}{R} \sin \frac{r}{R}+r\right),
\end{aligned}
$$

(3.4) becomes in this case

$$
\begin{aligned}
V_{r}=V & +\frac{1}{2}\left(r+R \cos \frac{r}{R} \sin \frac{r}{R}\right) \int_{M} d V_{M}+R^{2} \sin ^{2} \frac{r}{R} \int_{M} H d V_{M} \\
& +\frac{1}{2} R^{2}\left(r-R \cos \frac{r}{R} \sin \frac{r}{R}\right) \int_{M} k_{1} k_{2} d V_{M} .
\end{aligned}
$$

On the other hand, denoting the Gaussian curvature of $M$ by $K$, we have easily

$$
K=k_{1} k_{2}+\frac{1}{R^{2}} \text {. }
$$

Hence, by means of the Gauss-Bonnet theorem we obtain

$$
\begin{aligned}
\int_{M} k_{1} k_{2} d V_{M} & =\int_{M} K d V_{M}-\frac{1}{R^{2}} \int_{M} d V_{M}=2 \pi \cdot \chi(M)-\frac{1}{R^{2}} \int_{M} d V_{M} \\
& =4 \pi-\frac{1}{R^{2}} \int_{M} d V_{M},
\end{aligned}
$$

since $M$ is homeomorphic to $S^{2}$. Substituting this into the above equality, we obtain

$$
\begin{aligned}
V_{r}=V & +R \cos \frac{r}{R} \sin \frac{r}{R} \int_{M} d V_{M}+2 \pi R^{2}\left(r-R \cos \frac{r}{R} \sin \frac{r}{R}\right) \\
& +R^{2} \sin ^{2} \frac{r}{R} \int_{M} H d V_{M} .
\end{aligned}
$$


§4. An isoperimetric inequality for a convex domain in $S^{3}(R)$.

First of all, we investigate the integral in (2.11):

$$
\int_{F_{r}^{n-1}(y) \cap M} \frac{1}{\sqrt{1-\xi_{n} \xi_{n}}} d V_{F_{r}^{n-1}(y) \cap M} .
$$

For any point $x \in F_{r}^{n-1}(y) \cap M$ and a frame $\left(x, e_{1}, e_{2}, \cdots, e_{n}, e_{n+1}\right)$ as in $\S 1$, we have

$$
\begin{gathered}
\xi=\frac{1}{R \sin \frac{r}{R}}\left(y-x \cos \frac{r}{R}\right), \\
\xi_{i}=\left\langle\xi, e_{\imath}\right\rangle=\frac{y_{\imath}}{R \sin \frac{r}{R}}, \quad y_{i}=\left\langle y, e_{\imath}\right\rangle, \quad i=1,2, \cdots, n
\end{gathered}
$$

and

$$
y_{n+1}=\left\langle y, e_{n+1}\right\rangle=R \cos \frac{r}{R} .
$$

Along $\mathrm{F}_{r}^{n-1}(y) \cap M,\langle y, x\rangle=R^{2} \cos \frac{r}{R}$ implies

$$
\left\langle y, \sum_{\alpha} \omega_{\alpha} e_{\alpha}\right\rangle=\sum_{\alpha} y_{a} \omega_{\alpha}=0
$$

On the other hand, restricting the moving frame $\left(x, e_{1}, e_{2}, \cdots, e_{n+1}\right), x \in$ $F_{r}^{n-1}(y) \cap M$, to the one such that $e_{1}, \cdots, e_{n-2} \in T_{x}\left(F_{r}^{n-1}(y) \cap M\right)$, we have

$$
\omega_{n-1}=0 \text {, }
$$

and hence

$$
y_{1}=y_{2}=\cdots=y_{n-2}=0
$$

and

$$
y=y_{n-1} e_{n-1}+y_{n} e_{n}+R \cos \frac{r}{R} e_{n+1} .
$$

Using these relations, $d y=0$ implies

$$
\begin{gathered}
d y_{n-1}=y_{n} \omega_{n-1, n}, \quad d y_{n}=-y_{n-1} \omega_{n-1, n}, \\
y_{n-1} \omega_{\alpha, n-1}+y_{n} \omega_{a n}=\cos \frac{r}{R} \cdot \omega_{a}, \quad a=1, \cdots, n-2 .
\end{gathered}
$$

From (4.5) and the structure equation we obtain 


$$
\sum_{a=1}^{n-2} \omega_{n-1, a} \wedge \omega_{a}=0
$$

hence we can put

$$
\omega_{a, n-1}=\sum_{b=1}^{n-2} B_{a b} \omega_{b}, \quad B_{a b}=B_{b a} .
$$

$B_{a b}$ are the components of the 2nd fundamental form of $F_{r}^{n-1}(y) \cap M$ with respect to the normal unit vector $e_{n-1}$. By (4.4) and (4.7), we can put

$$
y_{n-1}=R \sin \frac{r}{R} \cos \theta, \quad y_{n}=R \sin \frac{r}{R} \sin \theta .
$$

Substituting these into (4.7) and (4.8), we get

$$
\omega_{n-1, n}=-d \theta \text {, }
$$

$$
\begin{gathered}
\cos \theta \cdot B_{a b}+\sin \theta \cdot A_{a b}=\frac{1}{R} \cot \frac{r}{R} \cdot \delta_{a b}, \\
a, b=1,2, \cdots, n-2 .
\end{gathered}
$$

From the equality :

$$
\omega_{n-1, n}=\sum_{a=1}^{n-2} A_{n-1, a} \omega_{a} \quad \text { along } \quad F_{r}^{n-1}(y) \cap M
$$

and (4.11), we can put

$$
A_{n-1, a}=-\nabla_{e_{a}} \theta,
$$

where $\nabla$ denotes the covariant derivation of $F_{r}^{n-1}(y) \cap M$.

Now, we suppose $n=3$ in the following. Then, $F_{r}^{2}(y) \cap M$ is composed of curves in general. Setting $\omega_{1}=d s,(4.11)$ and (4.13) imply

$$
\theta=-\int A_{12} d s+\text { const. }
$$

We have also

$$
\frac{d V_{F_{r}^{2}(y) \cap M}}{\sqrt{1-\xi_{3} \xi_{3}}}=\frac{d s}{\sqrt{1-\sin ^{2} \theta}}=\frac{d s}{\cos \theta}=-\frac{d \theta}{A_{12} \cos \theta} .
$$

In this case, (4.12) becomes

$$
B_{11} \cos \theta+A_{11} \sin \theta=\frac{1}{R} \cot \frac{r}{R},
$$

from which we get

$$
\cos \theta=\frac{1}{A_{11}{ }^{2}+B_{11}{ }^{2}}\left\{\frac{B_{11}}{R} \cot \frac{r}{R}+A_{11} \sqrt{A_{11}{ }^{2}+B_{11}{ }^{2}-\frac{1}{R^{2}} \cot ^{2} \frac{r}{R}}\right\} .
$$


Along the curve $F_{r}^{2}(y) \cap M$, we have

$$
\frac{d e_{1}}{d s}=B_{11} e_{2}+A_{11} e_{3}-\frac{1}{R} e_{4}
$$

and hence its curvature as a curve in $R^{4}$ is

$$
k(s)=\left|\frac{d e_{1}}{d s}\right|=\sqrt{B_{11}^{2}+A_{11}^{2}+\frac{1}{R^{2}}} .
$$

Using $k(s)$, the right-hand side of the above expression of $\cos \theta$ can be written as

$$
\cos \theta=\frac{1}{A_{11}{ }^{2}+B_{11}{ }^{2}} \cdot \frac{1}{R \sin \frac{r}{R}}\left\{B_{11} \cos \frac{r}{R}+A_{11} \sqrt{k^{2} R^{2} \sin ^{2} \frac{r}{R}-1}\right\} .
$$

Then, we have the following theorem which will be proved in $\S 5$.

THEOREM 2. Let $\Omega \subset S^{3}(R)$ be convex and for $\partial \Omega=M$ its normal curvature $A$ with respect to the inner normal unit vector satisfy $A_{0} \leqq A \leqq A_{1}$. Then, supposing $0<r<\pi R / 2$, for $\cos \theta$ given by (4.18) there exists a constant $C_{0}$ depending only on $A_{0}, A_{1}$ and $r$ such that $1 / \cos \theta \geqq C_{0} k(s)$.

Now, for a domain $\Omega$ in $S^{3}(R)$, let $r_{i}\left(r_{e}\right)$ be the supremum (infinimum) of radius of 3 -disk included in (containing) $\Omega$. Then, we have

THEOREM 3. Let $\Omega$ be a convex domain of $S^{3}(R)$ with a smooth boundary $\partial \Omega=M$. Let $H$ be the mean curvature of $M$. Then, for a fixed number $r\left(r_{i} \leqq r\right.$ $\leqq r_{e}$ ) we have

$$
\begin{aligned}
& R \cos \frac{r}{R} \sin \frac{r}{R}\left(\frac{2 R}{C_{0}} \tan \frac{r}{R}-1\right) \text { area }(M) \\
& \quad \geqq \operatorname{vol}(\Omega)+2 \pi R^{2}\left(r-R \cos \frac{r}{R} \sin \frac{r}{R}\right)+R^{2} \sin ^{2} \frac{r}{R} \int_{M} H d V_{M} .
\end{aligned}
$$

Proof. Since $\Omega$ is convex, we have easily $r_{e} \leqq \pi R / 2$. Therefore, we can utilize Theorem 2 for the domain $\Omega$. For a general point $y \in S^{3}(R)$, let $n(y)$ be the number of the components of $F_{r}^{2}(y) \cap M$. Let $C$ be one of them. For the curvature $k(s)$ of $C$ as a curve in $R^{4}$, we have $\int_{0}^{L} k(s) d s \geqq 2 \pi$ by the FenchelBorsuk theorem, where $L=\operatorname{length}(C)$. It is clear that the set of general points $y$ is open and dense in $S^{3}(R)$, and the function $n(y)$ is lower semi-continuous. Therefore, the set of $y$ with $n(y)=m$ is measurable with respect to the 3dimensional measure of $S^{3}(R)$ for any integer $m$. Setting

$$
F_{m}:=\operatorname{vol}\left(\left\{y \mid y \in S^{3}(R), n(y)=m\right\}\right),
$$


we obtain from Theorem 1 with $n=3$

$$
\begin{aligned}
& R^{2} \sin ^{2} \frac{r}{R} \cdot 4 \pi \cdot \operatorname{area}(M)=\int_{S^{3}(R)}\left(\int_{F_{r}^{2}(y) \cap M} \frac{d V_{F^{2}(y) \cap M}}{\sqrt{1-\xi_{3} \xi_{3}}}\right) d V_{S}(y) \\
& \quad \geqq C_{0} \int_{S^{3}(R)}\left(\int_{F_{r}^{2}(y) \cap M} k_{F_{r}^{2}(y) \cap M}(s) d s\right) d V_{S}(y) \\
& \quad \geqq 2 \pi C_{0} \int_{S^{3}(R)} n(y) d V_{S}(y)=2 \pi C_{0}\left(F_{1}+2 F_{2}+3 F_{3}+\cdots\right),
\end{aligned}
$$

i. e.

$$
\frac{2 R^{2}}{C_{0}} \cdot \sin ^{2} \frac{r}{R} \cdot \operatorname{area}(M) \geqq F_{1}+2 F_{2}+3 F_{3}+\cdots
$$

On the other hand, we see easily that

$$
\begin{aligned}
\Omega_{r}: & =\left\{x \mid x \in S^{3}(R), \operatorname{dis}_{S^{3}(R)}(x, \Omega)<r\right\} \\
& =\left\{y \mid n(y)>0, y \in S^{3}(R)\right\} \quad(\text { except a set of measure } 0)
\end{aligned}
$$

and

$$
V_{r}=\operatorname{vol}\left(\Omega_{r}\right)=F_{1}+F_{2}+F_{3}+\cdots .
$$

From (4.20) and (4.21), we have

$$
\frac{2 R^{2}}{C_{0}} \cdot \sin ^{2} \frac{r}{R} \cdot \operatorname{area}(M)-V_{r}=F_{2}+2 F_{3}+3 F_{4}+\cdots \geqq 0,
$$

and furthermore using (3.6) we obtain

$$
\begin{aligned}
& \frac{2 R^{2}}{C_{0}} \cdot \sin ^{2} \frac{r}{R} \cdot \operatorname{area}(M) \geqq V_{r}=\operatorname{vol}(\Omega)+R \cos \frac{r}{R} \sin \frac{r}{R} \cdot \operatorname{area}(M) \\
& \quad+2 \pi R^{2}\left(r-R \cos \frac{r}{R} \sin \frac{r}{R}\right)+R^{2} \sin ^{2} \frac{r}{R} \int_{M} H d V_{M},
\end{aligned}
$$

which is equivalent to (4.19).

q. e. d.

\section{§5. Proof of Theorem 2.}

According to the formula (4.18), we have

$$
\begin{aligned}
k(s) \cos \theta= & \sqrt{{A_{11}{ }^{2}+B_{11}{ }^{2}+\frac{1}{R^{2}}}_{1}} \cdot \frac{1}{{A_{11}{ }^{2}+B_{11}{ }^{2}}^{2}} \\
& \times\left\{B_{11} \frac{1}{R} \cot \frac{r}{R}+A_{11} \sqrt{{A_{11}{ }^{2}+B_{11}{ }^{2}-\frac{1}{R^{2}} \cot ^{2} \frac{r}{R}}^{R}}\right.
\end{aligned}
$$


and setting $A_{11}=A, B_{11}=B=u$ for simplicity we consider the following function of $u$

$$
f(u):=\frac{\sqrt{A^{2}+\frac{1}{R^{2}}+u^{2}}}{A^{2}+u^{2}}\left\{\frac{u}{R} \cot \frac{r}{R}+A \sqrt{A^{2}-\frac{1}{R^{2}} \cot ^{2} \frac{r}{R}+u^{2}}\right\} .
$$

Since $\Omega$ is convex, $A_{11} \geqq 0$ everywhere on $M=\partial \Omega$. We shall try to find an upper bound of $f(u)$ for $u \geqq 0$.

First of all, we write the right-hand side of (5.1) as

$$
f(u)=\frac{\sqrt{A^{2}+\frac{1}{R^{2}}+u^{2}}}{\sqrt{A^{2}+u^{2}}}\left\{\frac{1}{R} \cot \frac{r}{R} \frac{u}{\sqrt{A^{2}+u^{2}}}+\frac{A \sqrt{A^{2}-\frac{1}{R^{2}} \cot ^{2} \frac{r}{R}+u^{2}}}{\sqrt{A^{2}+u^{2}}}\right\} .
$$

We can easily see that the function $\frac{\sqrt{A^{2}+\frac{1}{R^{2}}+u^{2}}}{\sqrt{A^{2}+u^{2}}}$ is decreasing, $\frac{u}{\sqrt{A^{2}+u^{2}}}$ and $\frac{\sqrt{A^{2}-\frac{1}{R^{2}} \cot ^{2} \frac{r}{R}+u^{2}}}{\sqrt{A^{2}+u^{2}}}$ are increasing for $u \geqq 0$. Hence we have

$$
1<\frac{\sqrt{A^{2}+\frac{1}{R^{2}}+u^{2}}}{\sqrt{A^{2}+u^{2}}} \leqq \frac{\sqrt{A^{2}+\frac{1}{R^{2}}}}{A}, \quad 0 \leqq \frac{u}{\sqrt{A^{2}+u^{2}}}<1
$$

and

$$
\frac{\sqrt{A^{2}-\frac{1}{R^{2}} \cot ^{2} \frac{r}{R}}}{A} \leqq \frac{\sqrt{A^{2}-\frac{1}{R^{2}} \cot ^{2} \frac{r}{R}+u^{2}}}{\sqrt{A^{2}+u^{2}}}<1 \quad \text { for } \quad u \geqq 0 .
$$

Thus, we obtain

$$
f(u)<\frac{\sqrt{A^{2}+\frac{1}{R^{2}}}}{A}\left(\frac{1}{R} \cot \frac{r}{R}+A\right):=h(A) .
$$

The function $h(A)$ of $A$ has the properties as follows:

$$
\lim _{A \rightarrow+0} h(A)=\lim _{A \rightarrow+\infty} h(A)=+\infty
$$

and

$$
\frac{h^{\prime}(A)}{h(A)}=\frac{1}{A\left(A+\frac{1}{R} \cot \frac{r}{R}\right)\left(A^{2}+\frac{1}{R^{2}}\right)}\left(A^{3}-\frac{1}{R^{3}} \cot \frac{r}{R}\right),
$$


hence

$$
\begin{aligned}
& h^{\prime}(A)<0 \quad \text { for } \quad 0<A<\frac{1}{R}\left(\cot \frac{r}{R}\right)^{1 / 3}, \\
& h^{\prime}(A)>0 \quad \text { for } \quad \frac{1}{R}\left(\cot \frac{r}{R}\right)^{1 / 3}<A .
\end{aligned}
$$

Let us suppose from the convexity of $\Omega$ that

$$
(0<) A_{0} \leqq A \leqq A_{1} .
$$

Setting

$$
\max \left(h\left(A_{0}\right), h\left(A_{1}\right)\right)=\frac{1}{C},
$$

we have

$$
f(u)<h(A) \leqq \frac{1}{C},
$$

that is

$$
\frac{1}{\cos \theta}>C k(s) \text {. }
$$

q.e.d.

In the following, we shall show that $C$ in (5.4) can be replaced with a more sharper constant $C_{0}$. Setting

$$
\left\{\begin{array}{l}
f_{1}(u):=\frac{u}{A^{2}+u^{2}} \sqrt{A^{2}+\frac{1}{R^{2}}+u^{2}}, \\
f_{2}(u):=\frac{1}{A^{2}+u^{2}} \sqrt{A^{2}+\frac{1}{R^{2}}+u^{2}} \sqrt{A^{2}-\frac{1}{R^{2}} \cot ^{2} \frac{r}{R}+u^{2}},
\end{array}\right.
$$

we write $f(u)$ as

$$
f(u)=\frac{1}{R} \cot \frac{r}{R} \cdot f_{1}(u)+A f_{2}(u) .
$$

First of all, since we have

$$
f_{1}^{\prime}(u)=\frac{1}{\left(A^{2}+u^{2}\right)^{2} \sqrt{A^{2}+\frac{1}{R^{2}}+u^{2}}}\left\{A^{2}\left(A^{2}+\frac{1}{R^{2}}\right)+\left(A^{2}-\frac{1}{R^{2}}\right) u^{2}\right\},
$$

we obtain easily the following: 
i) when $A<\frac{1}{R}, \quad f_{1}(u) \leqq f_{1}\left(A \sqrt{\frac{1+R^{2} A^{2}}{1-R^{2} A^{2}}}\right)=\frac{1+R^{2} A^{2}}{2 A R} \quad$ for $\quad u \geqq 0$;

ii) when $A \geqq \frac{1}{R}, \quad f_{1}(u)$ is monotone increasing and so

$$
f_{1}(u)<\lim _{u \rightarrow+\infty} f_{1}(u)=1 \quad \text { for } \quad u \geqq 0 .
$$

Second, we have

$$
\begin{aligned}
f_{2}^{\prime}(u)= & \frac{u}{\left(A^{2}+u^{2}\right)^{2} \sqrt{A^{2}+\frac{1}{R^{2}}+u^{2}} \sqrt{A^{2}-\frac{1}{R^{2}} \cot ^{2} \frac{r}{R}+u^{2}}} \times \\
& \times\left\{-\frac{A^{2}}{R^{2}}\left(1-\cot ^{2} \frac{r}{R}\right)+\frac{2}{R^{4}} \cot ^{2} \frac{r}{R}-\frac{1}{R^{2}}\left(1-\cot ^{2} \frac{r}{R}\right) u^{2}\right\} .
\end{aligned}
$$

Hence we have the following:

a) Case $0<r \leqq \frac{\pi}{4} R, f_{2}(u)$ is monotone increasing and so

$$
f_{2}(u)<\lim _{u \rightarrow+\infty} f_{2}(u)=1 \quad \text { for } \quad u \geqq 0 \text {, }
$$

and

b) Case $\frac{\pi}{4} R<r \leqq \frac{\pi}{2} R$, from the equation

$$
-\frac{A^{2}}{R^{2}}\left(1-\cot ^{2} \frac{r}{R}\right)+\frac{2}{R^{4}} \cot ^{2} \frac{r}{R}=0,
$$

we obtain $A=\frac{\sqrt{2}}{R \sqrt{\tan ^{2} \frac{r}{R}-1}}$, and so

i) when $0<A<\frac{\sqrt{2}}{R \sqrt{\tan ^{2} \frac{r}{R}-1}}, f_{2}(u) \leqq f_{2}\left(\frac{1}{R} \sqrt{\frac{2-A^{2} R^{2}\left(\tan ^{2} \frac{r}{R}-1\right)}{\tan ^{2} \frac{r}{R}-1}}\right)$

$$
=\frac{1}{\sin \frac{2 r}{R}} \quad \text { for } \quad u \geqq 0 \text {, }
$$


ii) when $A \geqq \frac{\sqrt{2}}{R \sqrt{\tan ^{2} \frac{r}{R}-1}}, f_{2}(u)$ is monotone decreasing and so

$$
f_{2}(u) \leqq f_{2}(0)=\frac{\sqrt{A^{2} R^{2}+1} \sqrt{A^{2} R^{2}-\cot ^{2}-\frac{r}{R}}}{A^{2} R^{2}} \text { for } u \geqq 0 .
$$

On the other hand, we compare the separating values $\frac{\sqrt{2}}{R \sqrt{\tan ^{2} \frac{r}{R}-1}}$ and $\frac{1}{R}$ for $A^{\boldsymbol{x}}$ with respect to $f_{2}(u)$ and $f_{1}(u)$ respectively. We see easily that

$$
\frac{\sqrt{2}}{R \sqrt{\tan ^{2} \frac{r}{R}-1}} \begin{cases}>\frac{1}{R} & \text { for } \frac{\pi}{4} R<r<\frac{\pi}{3} R, \\ =\frac{1}{R} & \text { for } \quad r=\frac{\pi}{3} R, \\ <\frac{1}{R} & \text { for } \quad \frac{\pi}{3} R<r \leqq \frac{\pi}{2} R .\end{cases}
$$

From the above arguments, we define the following functions $h_{\imath}(A), \imath=1$, 2,3 , as follows :

1) Case $0<r \leqq \frac{\pi}{4} R$,

$$
h_{1}(A):= \begin{cases}\frac{1+A^{2} R^{2}}{2 A R^{2}} \cot \frac{r}{R}+A & \text { for } 0<A<\frac{1}{R}, \\ \frac{1}{R} \cot \frac{r}{R}+A & \text { for } A \geqq \frac{1}{R} ;\end{cases}
$$

2) Case $\frac{\pi}{4} R<r \leqq \frac{\pi}{3} R$,

$$
h_{2}(A):=\left\{\begin{array}{l}
\frac{1+A^{2} R^{2}}{2 A R^{2}} \cot \frac{r}{R}+\frac{A}{\sin \frac{2 r}{R}} \quad \text { for } 0<A<\frac{1}{R}, \\
\frac{1}{R} \cot \frac{r}{R}+\frac{A}{\sin \frac{2 r}{R}} \quad \text { for } \quad \frac{1}{R} \leqq A<\frac{\sqrt{2}}{R \sqrt{\tan ^{2} \frac{r}{R}-1}},
\end{array}\right.
$$




$$
\begin{aligned}
& \frac{r}{R} \cot \frac{1}{R}+\frac{\sqrt{A^{2} R^{2}+1} \sqrt{A^{2} R^{2}-\cot ^{2} \frac{r}{R}}}{A R^{2}} \\
& \text { for } \frac{\sqrt{2}}{R \sqrt{\tan ^{2} \frac{r}{R}-1}} \leqq A
\end{aligned} ;
$$

3) Case $\frac{\pi}{3} R<r \leqq \frac{\pi}{2} R$,

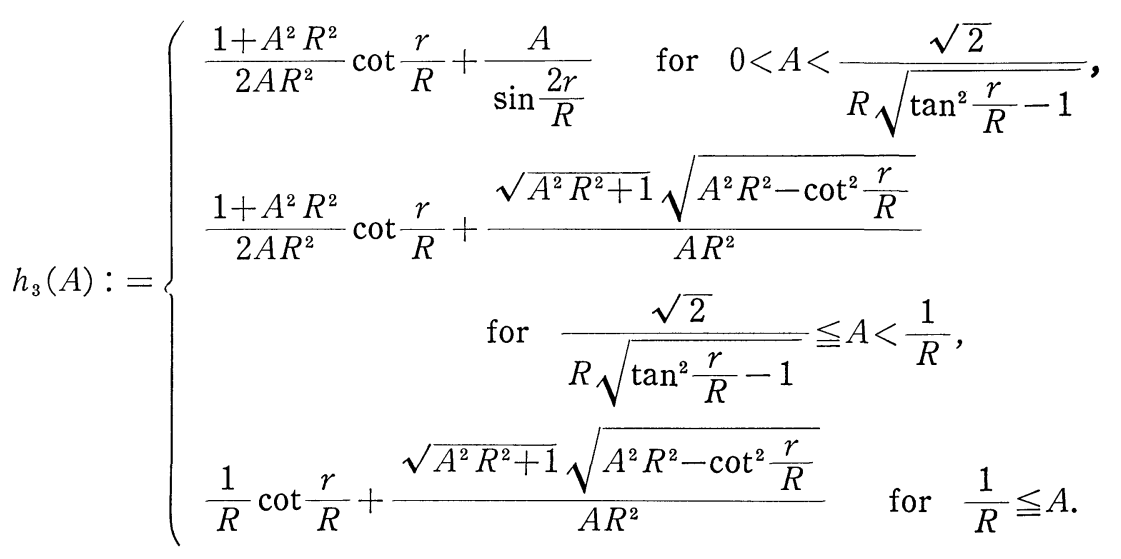

For each cases, we obtain from (5.7)

$$
f(u) \leqq h_{i}(A) \quad \text { for } \quad u \geqq 0 .
$$

Furthermore, we can prove easily that

1) Case $0<r \leqq \frac{\pi}{4} R, h_{1}(A)$ takes its minimum value at

$$
A_{1}^{*}=\frac{1}{R \sqrt{2 \tan \frac{r}{R}+1}}<\frac{1}{R}
$$

and it is monotone decreasing in $\left[0, A_{1}^{*}\right]$ and increasing in $\left[A_{1}^{*}, \infty\right)$;

2) Case $\frac{\pi}{4} R<r \leqq \frac{\pi}{3} R, h_{2}(A)$ takes its minimum value at

$$
A_{2}^{*}=\frac{1}{R \sqrt{1+\sec ^{2} \frac{r}{R}}}<\frac{1}{R}
$$

and it is monotone decreasing in $\left[0, A_{2}^{*}\right]$ and increasing in $\left[A_{2}^{*}, \infty\right)$; 
3) Case $\frac{\pi}{3} R<r \leqq \frac{\pi}{4} R, h_{3}(A)$ has the same property as $h_{2}(A)$.

Thus, making use of these functions $h_{\imath}(A), \imath=1,2,3$, for these three cases, we set

$$
\max \left\{h_{\imath}\left(A_{0}\right), h_{\imath}\left(A_{1}\right)\right\}=\frac{1}{C_{0}} .
$$

Then we have

$$
f(u)<h_{\imath}(A) \leqq \frac{1}{C_{0}} \quad \text { for } \quad A_{0} \leqq A \leqq A_{1} .
$$

It is clear that this $C_{0}$ is more sharper than $C$ for our purpose.

Tokyo Institute of Technology 